\title{
First-Order Signals in Compact QED with Monopole Suppressed Boundaries $^{\dagger}$
}

\author{
$\underline{\text { Th. Lippert }}^{\mathrm{a}}$, A. Bode $^{\mathrm{a}}$, V. Bornyakov ${ }^{\mathrm{b}}$, and K. Schillinga,c \\ ${ }^{a}$ Department of Physics, University of Wuppertal, D-42097 Wuppertal, Germany \\ bInstitute of High Energy Physics, 142284 Protvino, Russia \\ ${ }^{\mathrm{c}} \mathrm{HLRZ}$ c/0 KFA, D-52425 Jülich, Germany
}

Pure gauge compact QED on hypercubic lattices is considered with periodically closed monopole currents suppressed. We compute observables on sublattices which are nested around the centre of the lattice in order to locate regions where translation symmetry is approximately recovered. Our Monte Carlo simulations on $24^{4}-$ lattices give indications for a first-order nature of the $\mathrm{U}(1)$ phase transition.

\section{INTRODUCTION}

The presence of metastabilities at the phase transition of compact QED became obvious in simulations on $6^{4}$-lattices with periodic boundary conditions (p.b.c.)[1]. With increasing lattice size, there was growing confidence that the $\mathrm{U}(1)$ phase transition is exhibiting a weakly firstorder nature, culminating in the observation of extremely strong hysteresis effects appearing in Monte Carlo series on $16^{4}$-lattices[2].

This widely accepted opinion has been questioned recently. The authors of Refs. [3, 仼 have implemented compact $\mathrm{U}(1)$ on a lattice with trivial homotopy group, i.e. $\mathrm{SH}_{4}$, the surface of $H_{5}$. No indications for metastabilities have been found at $\beta_{c}$ in simulations on lattices of different sizesup to a number of degrees of freedom equivalent to a $16^{4}$-lattice with hyper-toroidal topology. Similarly, fixed boundary conditions (f.b.c.) hint at a continuous transition $[5.6$.

The implementation of U(1) on $\mathrm{SH}_{4}$ as well as with f.b.c. explicitly breaks the translation symmetry and produces inhomogenities of the free energy. One might argue that the absence of first-order effects can be explained in these terms. Alternatively it has been suggested in Ref. [4] that the existence of topologically non-

\footnotetext{
†Work supported by the Deutsche Forschungsgemeinschaft under grant No. Schi 257/3-2, Schi 257/1-4 and by EC contract CHRX-CT92-0051.
}

trivial monopole loops wrapping around the lattice due to p.b.c. might lead to a first-order signature at $\beta_{c}$.

We can check this assumption by studying the model with p.b.c. but periodically closed monopole loops forbidden and looking for firstorder signals. For this purpose, we suppress monopole currents emerging in the Monte Carlo update that would point outside the boundary shell and thus would be closed due to p.b.c.

As monitoring quantity we measure the average plaquette on the hyper-surface of subcubes nested around the centre of the lattice and we try to establish a region where translation symmetry is recovered approximately.

\section{SUPPRESSION OF MONOPOLES ON BOUNDARY}

We suppress monopoles on the boundaries in such a way that spinwaves can penetrate them. The monopole current is defined by [7]

$$
\begin{aligned}
m_{\mu}(x) & =\frac{1}{2} \epsilon_{\mu \nu \rho \sigma}\left[n_{\rho \sigma}(x+\nu)-n_{\rho \sigma}(x)\right], \\
n_{\rho \sigma} & =-2,-1,0,1,2
\end{aligned}
$$

where the number of Dirac sheets $n_{\mu \nu}(x)$ is given by the difference

$$
\frac{1}{2 \pi}\left[\bar{\Phi}_{\mu \nu}(x)-\Phi_{\mu \nu}(x)\right]
$$



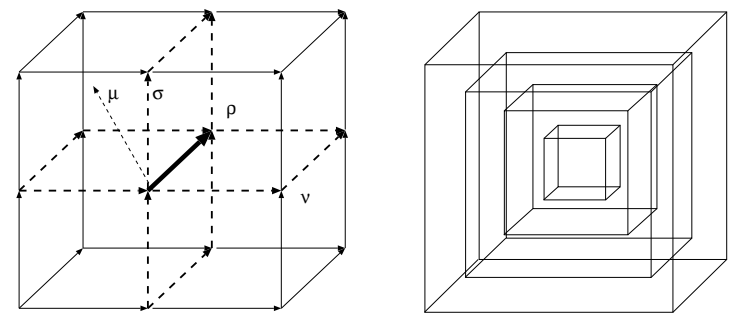

Figure 1. Plaquettes contributing to $m_{\mu}(x)$.

Figure 2. Measurements on nested shells.

between physical flux $\bar{\Phi}_{\mu \nu}(x)$ and plaquette flux $\Phi_{\mu \nu}(x)$ [7]. For a link $U_{\rho}$, to be updated in a boundary-cube with normalvector $e_{\mu}$, we search for the plaquettes associated with the Dirac sheets $n_{\rho \sigma}(x)$ that contribute to the monopole current $m_{\mu}(x)$ being parallel to $e_{\mu}$. These plaquettes are depicted in Fig. 11 with dotted lines. If, for any of the corresponding cubes, the change in flux is larger than $2 \pi$, the proposed link change is rejected in the Monte Carlo. This particular update rule fulfills detailed balance and is equivalent to an infinitely large chemical potential for the monopoles on the boundary shell[8] with the action $S=S_{W}+S_{\lambda} . S_{W}$ is the standard Wilson action and $S_{\lambda}$ is the additional term which suppresses monopole currents $m_{\mu}$ at the lattice slice $x_{\mu}=0$ :

$S_{\lambda}=\lambda \cdot \sum_{\mu} \sum_{x: x_{\mu}=0}\left|m_{\mu}(x)\right|, \quad \lambda \rightarrow \infty$.

$S_{\lambda}$ explicitly breaks translation symmetry.

\section{RESULTS}

On the $16^{4}$ - as well as on the $24^{4}$-lattice, we first scanned the $\beta$-range in order to get a rough idea about the location of the (pseudo)-critical region. It is shifted towards smaller $\beta$-values as expected from monopole suppression. Table 1 shows the number of Metropolis sweeps we performed subsequently for the such chosen $\beta$-values and the plaquette averaged over the whole lattice. On the $24^{4}$-lattice in the average about $1 \%$ of the proposed updates are rejected at the boundary.

The plaquettes have been sampled on the surfaces of sub-hypercubes nested around the centre

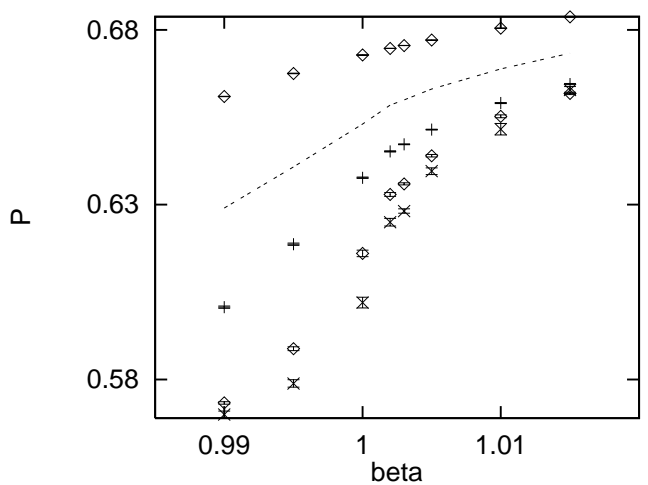

Figure 3. Average plaquette (dotted line) and plaquettes on shells $1,3,5$ and 7 (from top to bottom) on the $16^{4}$-lattice.

of the lattice. The 3D projection of these shells is shown in Fig. 2. The boundary shell is denoted by the index 1 , the inner shell gets the index $L / 2$.

Table 1: Lattice sizes, $\beta$-values and \# of sweeps.

\begin{tabular}{rrrr}
\hline size & $\beta$ & \# of sweeps & $\langle P\rangle$ \\
\hline $16^{4}$ & 0.990 & 30000 & $0.6290(2)$ \\
& 0.995 & 10000 & $0.6408(4)$ \\
1.000 & 60000 & $0.6530(2)$ \\
1.002 & 60000 & $0.6584(1)$ \\
1.003 & 60000 & $0.6599(1)$ \\
1.005 & 60000 & $0.6630(1)$ \\
1.010 & 10000 & $0.6688(2)$ \\
\hline $24^{4}$ & 1.0060 & 60000 & $0.6546(1)$ \\
1.0065 & 60000 & $0.6572(1)$ \\
1.0070 & 250000 & $0.65856(5)$ \\
1.007125 & 200000 & $0.65935(9)$ \\
1.0075 & 80000 & $0.66010(9)$ \\
1.0078 & 50000 & $0.66099(6)$ \\
1.0080 & 55000 & $0.66139(5)$
\end{tabular}

The boundary shell is always in a deconfined state. This is a well known phenomenon in the absence of monopoles 88]. Therefore, the variation of the plaquette value within the metastable region is very small for this shell. As the boundary shell contains the outweighting part of the sites, the plaquette average over the whole lattice is dominated by the contribution of the boundary. We found the influence of the boundary decreasing towards the centre, i.e. the plaquette values show increasing 'steps' (as functions of $\beta$ ) going to the inner shells, see Fig. 3 for the $16^{4}$-lattice. For inner shells the plaquette values are close to 


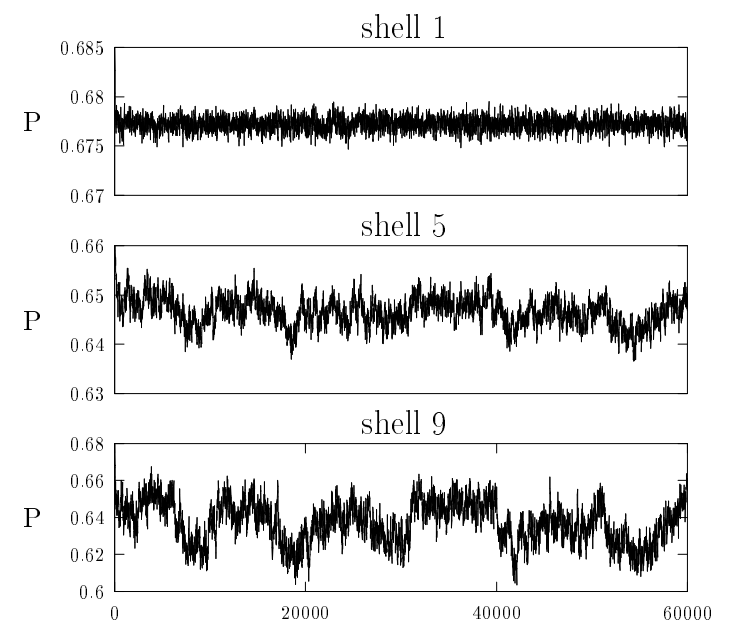

Figure 4. Time series on $24^{4}$-lattice for different shells.

each other and their behaviour is more and more like that of the plaquette of the $H_{4}$ system with p.b.c. We interpret this results as the onset of the recovery of translation invariance around the centre.

We carefully analyzed the time series for the inner shells on the $16^{4}$-lattice but the series merely exhibited a chaotic behaviour and no metastabilities could be identified. Therefore we decided to work on a very large $24^{4}$-system and we performed altogether nearly one million sweeps.

On this lattice, we found a much broader region in 4-d space at the centre where the behaviour of the system resembled that of $\mathrm{U}(1)$ on $H_{4}$ with p.b.c. and we found evidence for metastabilities in the time series. In Fig. (4, the time series of plaquettes for the shells 1,5 and 9 are depicted for $\beta=1.007125$. At this value of $\beta$ we encountered long metastabilities extending over more than 10000 sweeps as can be most clearly seen in the series of shell 9. As the fluctuations of the plaquette in inner shells are larger than the gap between the two metastable states we performed a windowing procedure in order to average out the fluctuations on small scales and applied the method to the time series of shell 9. The result is presented in Fig. 5, where we see a non-ambiguous double-peak structure of the histogram. More detailed results will be presented in a forthcoming

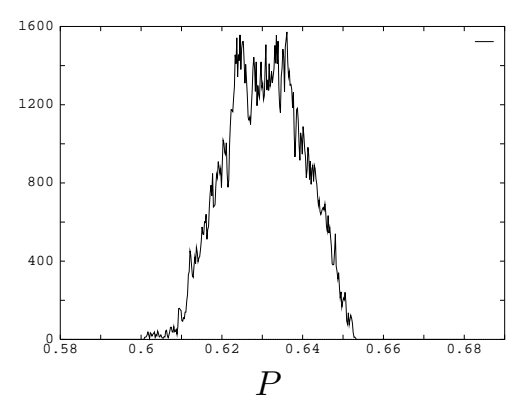

Figure 5. Histogram of shell 9 after removing of fluctuations.

paper.

\section{CONCLUSIONS}

In our simulations

- we did not observe first order signals on small lattices because of boundary effects. We believe this is true for f.b.c. as well.

- we observed metastabilities coming up on the $24^{4}$-lattice. This implies that periodically closed monopole loops can not be the reason for the first order nature of $\mathrm{U}(1)$ with p.b.c.

\section{REFERENCES}

1. J. Jersák, T. Neuhaus and P. M. Zerwas, Phys. Lett. No. 133B (1983) 103.

2. see e.g. Th. Lippert, G. Bhanot, K. Schilling and P. Ueberholz, Nucl. Phys. B (Proc. Suppl.) 30 (1993) 912.

3. Ch. Lang and T. Neuhaus, Nucl. Phys. B (Proc. Suppl.) 34 (1994) 543.

4. Ch. Lang and T. Neuhaus, Preprint Bielefeld University, 1994.

5. M. Baig and H. Fort, UAB-FT-338, 1994.

6. K.-H. Mütter and K. Schilling, Nucl. Phys. B 200 (1982) 362.

7. T. DeGrand and D. Toussaint, Phys. Rev. D22 (1980) 2478.

8. V. Bornyakov, V. Mitrjushkin and M. MüllerPreussker, Nucl. Phys. B (Proc. Suppl.) 30 (1993) 587. 